ORIGINAL RESEARCH

\title{
Mechanisms of Injury in Competitive Off-Road Bicycling
}

\author{
Tony K. Chow, MD; Robert L. Kronisch, MD \\ From the Department of Emergency Medicine, Loma Linda University Medical Center, Loma Linda, CA (Dr Chow), and the Student Health \\ Center, San Jose State University, San Jose, CA (Dr Kronisch).
}

\begin{abstract}
Objective.-To describe the mechanisms of injury from crashes during competitive off-road bicycling (mountain biking) and to examine the type, location, and severity of the resulting injuries.

Methods.-We examined and interviewed all bicyclists injured while competing at 7 off-road bicycling events. Information regarding the direction of fall and the presence of a collision or mechanical failure was obtained, and the injury patterns were compared.

Results.-There were 97 injured riders, with a mean age of 28.3 years. Most victims were male (74\%), and all cyclists wore helmets. Most injuries were minor and involved the extremities (70.5\%). Injuries sustained from falling forward over the handlebars occurred more often than from falling to the side $(65 \%$ vs $25 \%)$, tended to lead to injuries that were more severe (mean injury severity score $[\mathrm{ISS}]=3.4$ vs $1.7, P<.05)$, and produced more head and neck injuries $(56 \%$ vs $8 \%, P<.05)$. Falls to the side generally led to a lower extremity injury $(88 \%$ vs $57 \%, P<.05)$. Riders who were involved in collisions had injuries that were similar in severity and location to those of riders who had no collision.
\end{abstract}

Conclusions.- These findings suggest that off-road bicyclists whose mechanism of injury involves falling forward over the handlebars are at risk for more severe injury, especially to the head and neck.

Key words: off-road bicycling, mechanism of injury, trauma, injury

\section{Introduction}

Off-road bicycling, also known as mountain biking, has enjoyed increasing popularity as well as international attention since its beginnings in the 1970s. Today, mountain bikes, which are intended for use in any terrain, continue to be the volume leader in the bicycle industry, whose statistics show 55\% of bikes sold in 1997 were all-terrain bicycles. ${ }^{1}$ In spite of this dominance, information available on injury risks from off-road bicycling is relatively sparse and comes from the few epidemiological studies done recently. The surveys of recreational cyclists are consistent in their findings that off-road cycling often leads to injuries ( $51 \%$ to $86 \%$ within the past year), but most injuries are minor. ${ }^{2,3}$ Other research focused on competitive cyclists ${ }^{4,5}$ and also demonstrated a preponderance of minor injuries. These studies were descriptive, focused mainly on the in-

This study was presented at the 46th Annual Meeting of The American College of Sports Medicine, Seattle, WA, June 2 to 5, 1999, and the Summer Conference and Annual Meeting of the Wilderness Medical Society, Park City, UT, August 8 to 12, 2000.

Corresponding author: Tony K. Chow, MD, Loma Linda University Medical Center, Department of Emergency Medicine, A-108, 11234 Anderson St, Loma Linda, CA 92354 (e-mail: drtkchow@aol.com). juries themselves, and offered little information about the mechanism of injury.

The limitation with some of these studies is that they are retrospective in nature and depend on victim recall. Also, they include injuries of all types, most of which are minor, requiring no professional evaluation or time off from work or school. Kronisch et $\mathrm{al}^{5,6}$ studied competitive off-road cyclists in a prospective manner and described injury rates and patterns and mechanism of injury. The data suggested a possible correlation between injury mechanism and injury severity. This study was done to evaluate more victims of mountain bike racing in an effort to better describe the relationship between injury mechanism and injury type, location, and severity.

\section{Methods}

This investigation is an extension of the study begun in 1994 and now consists of a larger sample population. The methods have been described in detail previously 6 and are summarized here. Beginning in 1994, we attended a total of 7 mountain bike racing events organized by the National Off-Road Bicycling Association 
Table 1. Characteristics of crashes leading to injury among 97 victims in competitive off-road cycling, 1994-98

\begin{tabular}{|c|c|c|}
\hline \multirow[b]{2}{*}{ Characteristic } & \multicolumn{2}{|c|}{$\begin{array}{c}\text { Injured victims, } \\
\mathrm{n}=97\end{array}$} \\
\hline & $(N o)$. & $(\%)$ \\
\hline \multicolumn{3}{|l|}{ Grade } \\
\hline Downhill & 83 & 85.6 \\
\hline Flat & 8 & 8.2 \\
\hline Uphill & 3 & 3.1 \\
\hline N/A (observed trials) & 3 & 3.1 \\
\hline \multicolumn{3}{|l|}{ Cause of fall } \\
\hline Collision with stationary object & 7 & 7.2 \\
\hline Collision with another rider & 16 & 16.5 \\
\hline Loss of traction & 14 & 14.4 \\
\hline Loss of control & 31 & 32.0 \\
\hline Mechanical problem & 15 & 15.5 \\
\hline Other or unknown & 14 & 14.4 \\
\hline \multicolumn{3}{|l|}{ Direction of fall } \\
\hline Forward & 63 & 64.9 \\
\hline Side & 24 & 24.7 \\
\hline Other or unknown & 10 & 10.3 \\
\hline
\end{tabular}

(NORBA) - Vail and Mount Spokane in 1995 as well as Mammoth Mountain each summer from 1994 to 1998. Events included the following: 1) downhill racing, a high-speed individual time trial; 2) cross-country, an endurance event with uphill and downhill riding; 3) dual slalom, a short downhill course consisting of multiple turns, gates, and jumps in which 2 riders maneuver side by side; and 4) observed trials, an obstacle course that tests the cyclist's balancing skills. Participants included amateur as well as professional cyclists, and all were required to wear helmets.

All riders who sustained an injury severe enough to prevent them from continuing their respective race were eligible. Each race event provided emergency medical care, and one or both of the authors were available to evaluate the victims who fit the inclusion criteria. All diagnoses were provided by the evaluating physician at the scene or by the physician at the emergency department of local hospitals if victims required more sophisticated care. The abbreviated injury score ${ }^{7}$ was determined for each diagnosis, and the injury severity score (ISS) ${ }^{8,9}$ was then calculated.

Each victim was also asked to answer a standardized questionnaire that elicited information regarding demographics and mechanism of injury, including direction of fall, presence of collision, and mechanical failure. This information was provided by eye witnesses of the crash for racers who could not recall the event. All victims gave written consent to participate in this study, and
Table 2. Characteristics of injuries among 97 victims in competitive off-road cycling, 1994-98

\begin{tabular}{llr}
\hline \multicolumn{1}{c}{$\begin{array}{c}\text { Anatomic } \\
\text { location }\end{array}$} & \multicolumn{1}{c}{ Type of injury } & $\begin{array}{r}\text { No. }(\%) \\
\text { of all } \\
\text { injuries }\end{array}$ \\
\hline \multirow{2}{*}{ Head/neck } & Concussion & $13(6.8)$ \\
Face & Neck strain & $8(4.2)$ \\
& Fracture & $2(1.0)$ \\
Trunk & Laceration/abrasion & $16(8.4)$ \\
& Rib fracture & $4(2.1)$ \\
Back/spine & Chest wall contusion & $6(3.2)$ \\
& Fracture & $1(0.5)$ \\
Upper extremity & Strain & $1(0.5)$ \\
& Contusion/abrasion & $5(2.6)$ \\
& Sprain/strain & $23(12.1)$ \\
Lower extremity & Contusion/laceration/abrasion & $8(4.2)$ \\
& Sracture & $34(17.9)$ \\
& Sprain/strain & $4(2.1)$ \\
Total & Contusion/laceration/abrasion & $61(32.1)$ \\
& & 190 \\
\hline
\end{tabular}

the protocol was approved by the Institutional Review Board at San Jose State University.

All information obtained was entered into a computer program (MS Excel) and tabulated. We used the statistical program Primer of Biostatistics for calculation of the $P$ value from chi-square testing or Mann-Whitney rank sum where appropriate.

\section{Results}

We evaluated 97 victims whose ages ranged from 15 to 59 with a mean of 28.3 years. Most victims were male $(n=72)$. The majority of crashes occurred while riding downhill (85.6\%), and the most common direction of fall was forward $(64.9 \%)$. The cause of the crash varied widely, although most of the time, the victim cited loss of control as the cause. Collisions and mechanical failure were less common and occurred $23.7 \%$ and $15.5 \%$ of the time, respectively. These results are summarized in Table 1.

A total of 190 injuries were sustained by all victims, and these are shown in Table 2. The majority of injuries were minor, consisting of contusions, abrasions, and lacerations to soft tissue. Most injuries occurred to the extremities, with a nearly equal distribution to the upper and lower limbs (34.2\% vs $36.3 \%)$, but the incidence of fracture or dislocation was higher in the upper extremities (12.1\% vs $2.1 \%)$. Incidence of injury to the head, neck, and face was significant and included $20.5 \%$ of all 
Table 3. Comparison of injuries from falls forward vs falls to the side, 1994-98

\begin{tabular}{lccc}
\hline & Falls forward & Falls to the side & P-value \\
\hline $\begin{array}{l}\text { No. of injured riders } \\
\text { No. of victims requiring } \\
\quad \text { emergency department evaluation }\end{array}$ & 63 & 24 & .02 \\
Mean injury severity score & $38^{*}(60 \%) \dagger$ & $7(29 \%)$ & .001 \\
Injuries to head/face/neck & & & .1 .7 \\
Injuries to trunk & $35(56 \%)$ & $2(8 \%)$ & .001 \\
Injuries to back/spine & $9(14 \%)$ & $1(4 \%)$ & .34 \\
Injuries to upper extremity & $6(10 \%)$ & $16(67 \%)$ & .18 \\
Injuries to lower extremity & $48(76 \%)$ & $21(88 \%)$ & .53 \\
\end{tabular}

*Two of the 38 victims were hospitalized.

$\uparrow$ All percentage figures reflect percentage of injured riders in that category.

injuries. Forty-five riders (46.4\%) were treated onsite, and, of the 52 injured cyclists who were sent to local hospitals, 3 required hospital admission. The ISS ranged from 1 to 17 with a mean of 2.9. No fatalities occurred.

We analyzed the direction of fall and its relationship to the location and severity of injury. We directly compared these parameters as shown in Table 3. A fall forward over the handlebars was not only the most common direction of fall, but it also led to injuries that were more severe overall. Victims from falls forward were more likely to require emergency department evaluation $(60 \%$ vs $29 \%, P<.05)$ and had higher ISS $(3.4$ vs $1.7, P<$ $.05)$. The data demonstrate that the head, neck, and face are more prone to injury when cyclists fall forward rather than to the side $(56 \%$ vs $8 \%, P<.05)$. In fact, all victims who sustained a concussion did so from a fall forward. In contrast, falls to the side generally led to extremity trauma, with the lower limb being more vulnerable $(88 \%$ vs $57 \%, P<.05)$ in this type of fall.

We also examined the other aspects of injury mechanism and found that collisions led to injuries that were no more severe than injuries without a collision. In addition, the anatomic location of the injuries and types of injuries did not differ. Similarly, cyclists who cited loss of control, loss of traction, and mechanical failure did not sustain injuries that differed in location and severity from those of riders who did not report these mechanisms.

\section{Discussion}

The retrospective studies done to date on mountain bike injuries clearly demonstrated a high incidence of crashes in this sport. The definition used in some surveys ${ }^{2,3}$ was broad and allowed inclusion of all injuries; thus, it is not surprising that the majority of injuries were minor, requiring no professional medical attention and no time off from school or work. One study that examined in- juries from off-road bicycling victims who were evaluated in emergency departments also showed most injuries were abrasions, contusions, and lacerations. ${ }^{10}$ Our results are consistent with the findings of these earlier studies, namely, injuries from off-road bicycling occur frequently but are usually minor. Minor traumatic injuries are probably inevitable from crashes that are inherent to the sport. In this study, we intentionally focused on more severe injuries, defined as those that caused the rider to discontinue the race and seek medical attention, so that we could examine the mechanisms leading to such injuries.

A brief review of fatalities and more serious injuries from road bicycling shows that the mechanisms of injury in those crashes are unique to riding on paved terrain and therefore do not help elucidate the risks in off-road bicycling. For example, many of the crashes on paved terrain involve collisions with motor vehicles. ${ }^{11,12}$ Such collisions are uncommon in off-road bicycling because of the difference in the 2 environments. The few documented cases of a rider who was on a mountain bike and had a collision with a motor vehicle occurred on paved terrain, and this indeed led to more serious injuries. ${ }^{2}$ Low helmet use has also been demonstrated to be a major factor leading to head injuries, especially in children. ${ }^{13}$ In contrast, helmet use was mandatory among the riders studied in our investigation, and previous studies confirm that helmet use among mountain bikers is high, ranging from $80 \%{ }^{10}$ to $88 \% .^{3}$ Alcohol use was also noted among brain-injured victims from road bicycling ${ }^{11}$ but was shown to be low in one survey among injured riders on mountain bikes. ${ }^{2}$ Many of the factors that have been shown to increase risk for significant injury from bicycling on paved terrain probably do not apply to offroad riding. This led to our attempt to describe distinctly different risk factors in this sport. 
In our study, we focused on 3 aspects of the mechanism of injury: presence of a collision, mechanical failure, and direction of fall. As discussed above, severe injuries have been noted with conventional road bicycling injuries from collisions, but those frequently involved a motor vehicle. In the sport of alpine skiing, in which the participant also negotiates down a mountain slope, studies have shown fatalities and serious injuries are frequently the result of collisions. ${ }^{14}$ Our data do not suggest that collisions lead to injuries in off-road cyclists that are statistically more severe or that the location of injuries differs. Nor does mechanical failure appear to influence the location or severity of injury.

The only factor we identified that led to more serious injury was the direction of fall. This has been suggested from reports of head and facial injuries from riding downhill in off-road situations ${ }^{15}$ and from our previous investigation, which had fewer victims. ${ }^{6}$ Those bicyclists who fell forward were more likely to require an evaluation in the emergency department, and they also tended to have a higher ISS compared with those who fell to the side. Falls forward also led to a different distribution of injuries. Head injuries are the most serious type of injury from cycling and are common in studies of road bicycling. From our data, head injuries occurred only when the rider fell forward. This mechanism also led to facial injuries not seen in other falls. Falls to the side more commonly led to trauma to the lower extremities, whereas injury to other parts of the body were very uncommon.

These results suggest that future studies on increased safety would do well to focus on identifying situations in which the rider is prone to a forward fall. For example, Ettlinger et al ${ }^{16}$ reviewed the actual falls that resulted in anterior cruciate ligament sprains in injured alpine skiers and identified distinct features of such falls that led to an increased risk of tear to this ligament. They went further and developed a set of recommendations for professional skiers designed to train them to fall in such a way that reduced the incidence of anterior cruciate ligament tears. Similarly, there may exist a number of riding styles and positions that may lead the off-road cyclist to be in a vulnerable posture more prone to forward falls. Identifying and avoiding such risky positions may result in less serious injuries.

Although our present study examines competitive offroad bicyclists, we believe the information obtained is applicable to all off-road bicyclists, including recreational riders, because we focus on mechanisms and not rates of injury, as we did in previous investigations. 5,6 The overall distribution of injuries in our study compares similarly with those found in previously mentioned studies, $2,3,10$ which examined all off-road bicyclists without making specific references to the recreational vs competitive nature of the ride. The other common feature of all these studies is the finding that the majority of crashes leading to injury in off-road bicycling occur while riding downhill. Our data do not discern whether the increased velocity from downhill riding or the difference in center of gravity of the cyclist as a result of the downhill position is the more important factor leading to forward falls. Further studies are needed in this area.

\section{References}

1. National Bicycle Dealers Association. 1998-1999. Statpak: A Look at the Bicycle Industry's Vital Statistics. Newport Beach, CA: National Bicycle Dealers Association; 1998.

2. Chow TK, Bracker MD, Patrick K. Acute injuries from mountain biking. West J Med. 1993;159:145-148.

3. Kronisch RL, Rubin AL. Traumatic injuries in off-road bicycling. Clin J Sport Med. 1994;4:240-244.

4. Pfeiffer RP. Off-road bicycle racing injuries - the NORBA pro/elite category. Clin Sports Med. 1994;13:207-218.

5. Kronisch RL, Chow TK, Simon LM, Wong PF. Acute injuries in off-road bicycle racing. Am J Sports Med. 1996; 24:88-93.

6. Kronisch RL, Pfeiffer RP, Chow TK. Acute injuries in cross-country and downhill off-road bicycle racing. Med Sci Sports Exerc. 1996;28:1351-1355.

7. Association for the Advancement of Automotive Medicine. The Abbreviated Injury Scale. 1990 revision. Des Plaines, IL: Association for the Advancement of Automotive Medicine; 1990.

8. Baker SP, O'Neill B, Haddon W, Long WB. The injury severity score: a method for describing patients with multiple injuries and evaluating emergency care. $J$ Trauma. 1974;14:187-196.

9. Baker SP, O'Neill B. The injury severity score: an update. J Trauma. 1976;16:882-885.

10. Rivara FP, Thompson DC, Thompson RS, Rebolledo V. Injuries involving off-road cycling. J Fam Pract. 1997;44: 481-485.

11. Kraus JF, Fife D, Conroy C. Incidence, severity and outcomes of brain injuries involving bicycles. Am J Public Health. 1987;77:76-78.

12. Kruse DL, McBeath AA. Bicycle accidents and injuries, a random survey of a college population. Am J Sports Med. 1980;8:342-344.

13. Puranik S, Long J, Coffman S. Profile of pediatric bicycle injuries. South Med J. 1998;91:1033-1037.

14. Morrow PL, McQuillen EN, Eaton LA, Bernstein CJ. Downhill ski fatalities: the Vermont experience. J Trauma. 1988;28:95-100.

15. Chow TK, Corbett S, Farstad D. Do conventional helmets provide adequate protection in mountain biking? Wilderness Environ Med. 1995;6:385-390.

16. Ettlinger CF, Johnson RJ, Shealy JE. A method to help reduce the risk of serious knee sprains incurred in alpine skiing. Am J Sports Med. 1995;23:531-537. 